\title{
Chronic adolescent exposure to cannabis in mice leads to long-term sex-biased changes in gene expression networks across brain regions
}

\author{
Yanning Zuo ${ }^{1,2}$, Attilio lemolo ${ }^{5}$, Patricia Montilla-Perez ${ }^{5}$, Hairi Li $^{5}$, Xia Yang ${ }^{1,3,4}$, \\ Francesca Telese ${ }^{5}$ \\ ${ }^{1}$ Department of Integrative Biology and Physiology, UCLA \\ ${ }^{2}$ Neuroscience Interdepartmental Program, UCLA \\ ${ }^{3}$ Institute for Quantitative and Computational Biosciences, UCLA \\ ${ }^{4}$ Brain Research Institute, UCLA \\ ${ }^{5}$ Department of Medicine, University of California, San Diego, La Jolla, CA, 92093, USA \\ Corresponding authors: Francesca Telese, Ph.D. (ftelese@ucsd.edu)
}

\begin{abstract}
Background: The molecular mechanisms underlying the long-lasting behavioral changes associated with adolescent cannabis use are poorly understood. To this end, we performed gene network analyses of multiple brain regions in adult mice exposed during the entire adolescence to $\Delta$-9-tetrahydrocannabinol (THC), the major psychoactive component of cannabis.
\end{abstract}

Methods: Two weeks after the last exposure to THC (10 mg/kg) or vehicle, we measured cognitive behaviors and profiled the transcriptomes of 5 brain regions from 12 female and 12 male mice. We performed differential gene expression analysis and constructed gene coexpression networks (modules) to identify THC-induced transcriptional alterations at the level of individual genes, gene networks, and biological pathways. We integrated the THC-correlated modules with human traits from genomewide association studies to identify potential regulators of disease-associated networks. Results: THC impaired cognitive behaviors of mice, with memory being more impacted in females than males, which coincided with larger transcriptional changes in the female brain. Modules involved in endocannabinoid signaling and inflammation were correlated 
with memory deficits in the female dorsal medial striatum and ventral tegmental area, respectively. Converging pathways related to dopamine signaling and addiction were altered in the female amygdala and male nucleus accumbens. Moreover, the connectivity map of THC-correlated modules uncovered intra- and inter-region molecular circuitries influenced by THC. Lastly, modules altered by THC were enriched in genes relevant for human cognition and neuropsychiatric disorders.

Conclusions: These findings provide novel insights concerning the genes, pathways and brain regions underlying persistent behavioral deficits induced by adolescent exposure to THC in a sex-specific manner.

\section{Introduction}

Numerous studies have reported consistent and clear association of high frequency and dose of cannabis use with long-term adverse health effects, including higher risk of developing psychotic disorders, dependence, memory deficits, and anxiety ${ }^{1-5}$, posing a major public health concern for vulnerable populations, such as adolescents.

Adolescence is a critical developmental period for reproductive functions, brain maturation and the emergence of sex differences in social, cognitive, and emotional behaviors $^{6}$. The endocannabinoid (eCB) system plays an important role in brain maturation, and, as such, drug-induced alterations of the eCB system during adolescence could lead to substantial sex-specific behavioral alterations and increased risk for psychopathology in adulthood ${ }^{6-8}$. 
Although animal studies have recapitulated the impact of adolescent cannabis exposure on behavioral outcomes that are relevant to neuropsychiatric disorders, our knowledge of the underlying neurobiological processes remains limited. Long-lasting changes in gene expression and epigenetic marks are likely to be an important mechanism mediating drug-induced neuroadaptations ${ }^{9}$. However, limited data is available concerning sex-specific transcriptional responses to cannabis use across different brain regions ${ }^{10}$, limiting our ability to dissect brain region specificity and crossbrain networks in the female and male brain.

Multiple brain regions are involved in cognitive and reward processes that are linked to early and frequent cannabis use. These brain regions included the prefrontal cortex (PFC), which undergoes functional and structural remodeling during adolescence and mediates higher cognitive functions ${ }^{11}$; the nucleus accumbens (NAc), which drives motivation and reward behaviors ${ }^{12}$; the dorsal medial striatum (DMS), which plays a critical role in goal-directed responses ${ }^{13}$; amygdala (Amy), critical for processing emotions and memories ${ }^{14}$; ventral tegmental area (VTA), important for a range of learning behaviors related to reward and stressful experiences ${ }^{15}$. However, the analysis of global gene expression changes altered by adolescent exposure to THC is limited to PFC in male rats ${ }^{10}$. Thus, more research is needed to address this knowledge gap.

Here, we used female and male mice that were chronically administered THC during adolescence and were subjected to behavioral analysis in adulthood. Next, we measured gene expression changes by RNA sequencing (RNA-seq) in 5 brain regions that are involved in cognitive and addiction-related processes. We examined gene expression and gene network changes associated with adolescent exposure to THC for 
each sex and within and between brain regions. Lastly, we performed integrative analyses of coexpression networks affected by THC in mice with human cognition traitrelated genes from genome wide association studies (GWAS) to identify links between adolescent THC use and neuropsychiatric disorders.

\section{Methods}

Detailed descriptions of experimental design and methods are included in the Supplementary Methods. All methods used in this study have been published recently ${ }^{16-19}$. RNA-seq data was deposited on the GEO (accession GSE189821).

\section{Results}

THC administration during adolescence impairs cognitive behaviors in a sex-

\section{specific manner}

We measured several behaviors in adult mice of both sexes ( $n=12 /$ group/sex) with or without chronic adolescent exposure to THC (Fig 1A). We used six different objects test (6-DOT) to assess the long-term effects of adolescent exposure to THC on object recognition memory ${ }^{16,20}$. THC exposure during adolescence significantly reduced the absolute (D1) and relative (D2) discrimination indexes by $36 \%$ and $30 \%$, respectively, compared to vehicle control group (Fig 1B-C). Despite the lack of a significant interaction of sex with treatment, post-hoc analysis showed that the effect was stronger in females than males (Fig. 1B-C). Moreover, only THC-exposed female mice showed a significant $40 \%$ decrease in the exploration of the novel object compared to the vehicle group (Fig. 1D).

The behavioral alterations triggered by THC were specific for the cognitive components of the assay, as treatment had no detectable effect on the distance 
traveled during the habituation phase (Fig. Suppl. 1A) nor on the total amount of exploratory activity towards objects in familiarization or test phase (Fig. Suppl. 1B-C).

When we conducted the three-chamber interaction test, social preference was significantly decreased by $12 \%$ in both female and male mice exposed to THC during adolescence compared to the vehicle group (Fig. 1E).

Next, we examined anxiety-like behaviors and did not observe significant differences in the willingness of mice to explore open environments (Fig. Suppl. 1D). Finally, THC exposure was not associated with changes in body weight at the time of 
behavioral testing, excluding potential confounding effects on exploratory activity (Fig.

Supp. 1E).
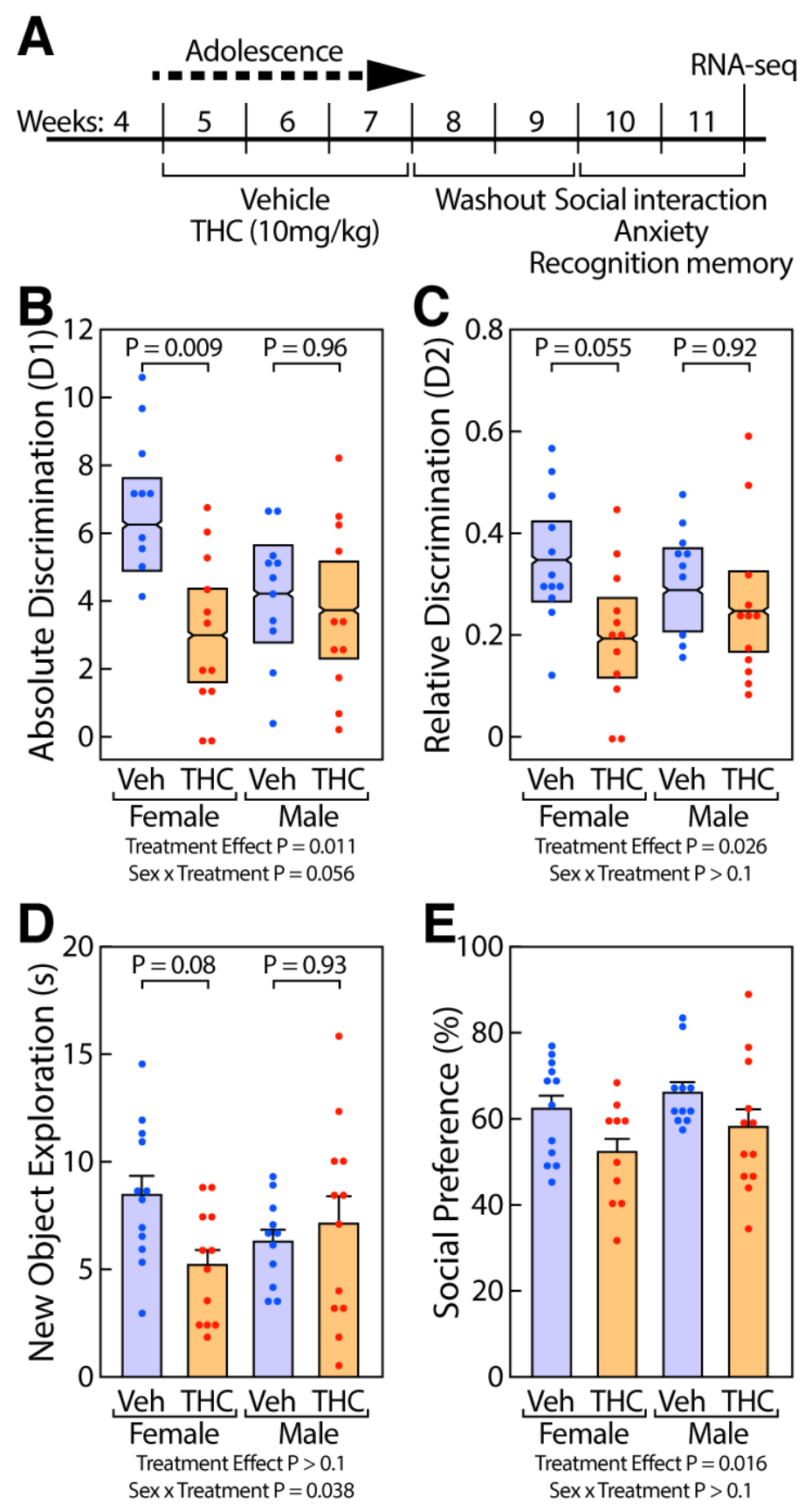

Figure 1: Adolescent exposure to THC reduced recognition memory and social interaction in a sex-specific manner. (A)

Timeline of the study design. (B)

D1 and (C) D2 are shown as mean $\pm 95 \%$ confidence intervals showing decreased recognition memory in THC-treated groups compared to vehicle controls.

Treatment effect $F_{1,41}=7.1, p=$ 0.011 for $\mathrm{D} 1$, and $\mathrm{F}_{1,42}=5.3, \mathrm{p}=$ 0.026 for D2. Sex x Treatment interaction $F_{1,41}=3.9, p=0.056$ for $\mathrm{D} 1$, and $\mathrm{F}_{1,42}=1.9, \mathrm{p}=0.176$ for D2. (D) Exploration time (s) of the novel object is expressed as mean \pm SEM showing a decrease only in female mice. Sex $x$ treatment interaction, $F_{1,42}=4.6$, $p=0.038$. (E) Social preference

$(\%)$ is expressed as mean \pm SEM and does not change across groups. Treatment effect $F_{1,41}, p=0.016$. $P$ values from Tukey HSD post-hoc analysis are shown in panel B-D. 
Overall, these results demonstrate that chronic exposure to THC during adolescence leads to long-term deficits in recognition memory and social interaction, with stronger memory deficits in female mice.

\section{Identification of DEGs associated with chronic adolescent exposure to THC}

We profiled the transcriptome of PFC, DMS, NAc, Amy and VTA from vehicle and THCtreated mice ( $n=6 /$ tissue/group/sex). PCA on the raw RNA-seq data showed that the studied brain regions can be distinguished based on their transcriptome, validating the accuracy of brain dissections (Fig. 2A).

We identified DEGs from each brain region in each sex by comparing THC and vehicle groups (Fig. 2B, Table S1). Using a cutoff of FDR $<0.1$ and $\operatorname{lgFC}>0.4$, we found the largest number of DEGs in the female Amy $(n=743)$ and in general more DEGs in females in most brain regions except NAc, which had the largest number of DEGs in males $(n=27)$. Similar trend was found at a more stringent cutoff of FDR $<0.05$. To explore the influence of sex on transcriptional responses to THC, we compared expression changes induced by THC treatment between males and females. At a statistical cutoff of FDR $<0.1$, this analysis yielded significant sex $x$ treatment interactions for DEGs in Amy ( $n=106)$, DMS $(n=7)$, and NAc $(n=13)$, but not for PFC and VTA (Fig. 2C, Suppl. Fig. 2), indicating that females and males responded differently to THC.

Based on the DEGs, we assessed the enrichment for biological pathways altered by THC (Fig. 2D-E, Table S2). In female Amy and DMS, DEGs showed enrichment for overlapping pathways, including addiction, opioid signaling and GPCR ligand binding. 
A

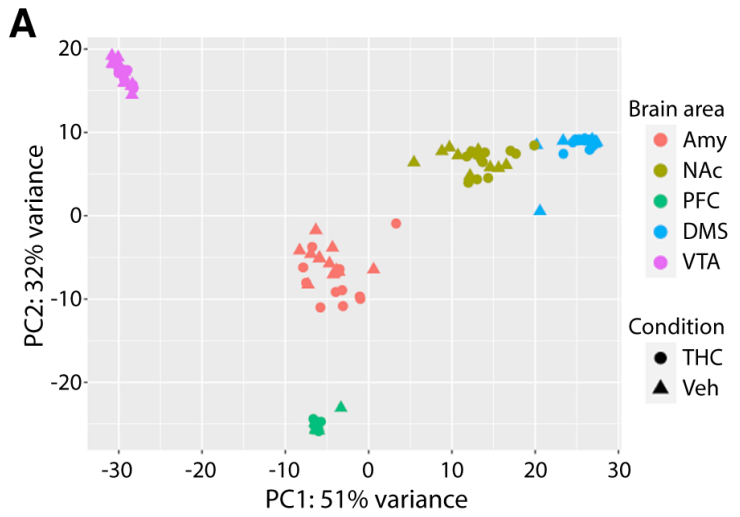

B

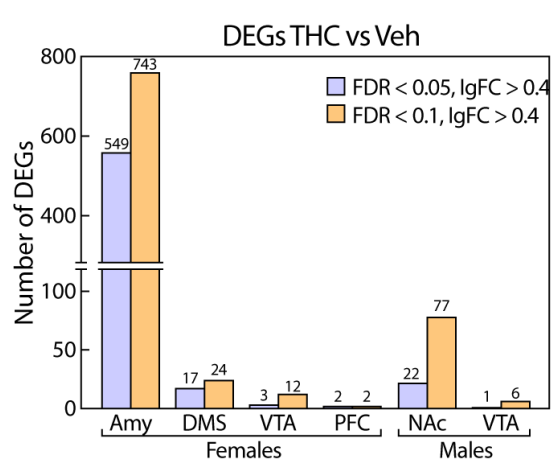

C SexxTreatment Interaction $(\mathrm{FDR}<0.1)$

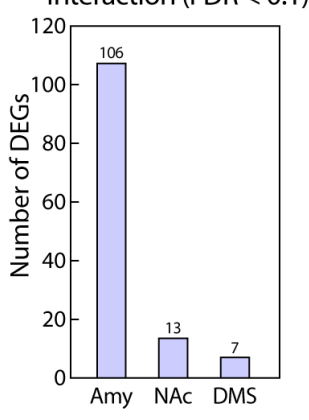

D

DEGs THC vs Veh

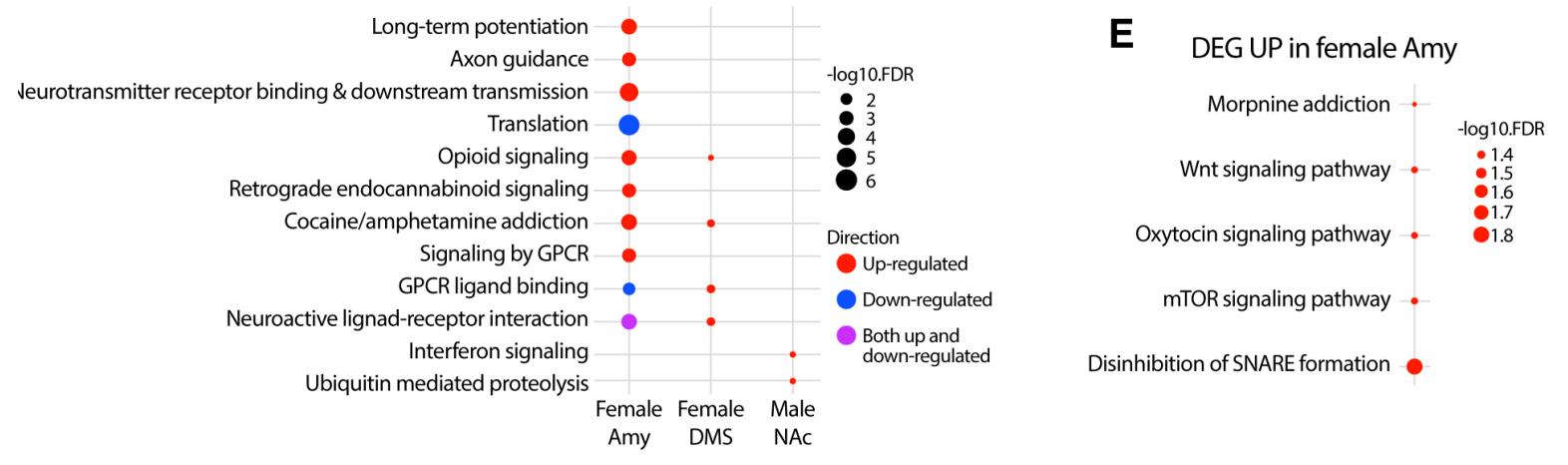

Figure 2: Adolescent THC exposure induced long-term sex-specific transcriptional changes. (A)

PCA visualization of male and female samples across brain regions and treatment conditions. (B) Number of DEGs across brain regions and sexes using different FDR cutoffs in analyses within each sex and brain region. (C) Number of DEGs for treatment by sex interaction across brain regions in analyses including both sexes for each brain region. (D) Pathway enrichment for DEGs in female Amy, DMS, and male NAc. Dot color depicts the direction of regulation and dot size illustrates the significance. (E) Upregulated treatment:sex interaction DEG pathway enrichment in female Amy. Dot size illustrates the significance. DMS, dorsomedial striatum; Amy, amygdala; NAc, nucleus accumbens; PFC, prefrontal cortex; VTA, ventral tegmental area; Veh, vehicle; DEG, differential expression gene.

Moreover, DEGs in female Amy were related to neurotransmission, axon guidance and retrograde cannabinoid signaling. Genes that were up-regulated only in females were linked to presynaptic SNARE complex formation and several signal transduction pathways (e.g. mTOR, Wnt, oxytocin signaling; Fig. 2E). In contrast, pathways associated with DEGs in male NAc did not overlap with those identified in females and were related to interferon signaling and ubiquitin-mediated proteolysis (Fig. 2D). 
In agreement with DEG analysis, threshold-free RRHO analysis showed that there was minimal overlap in DEGs when we compared gene expression changes between most pairs of brain regions or between sexes (Suppl. Fig. 3).

Together, these data provide strong evidence for sex-specific regulation of transcriptional responses by THC across several brain regions.

\section{Identification of gene coexpression networks correlated with THC treatment and cognitive traits.}

Because DEG analysis found limited significant changes at the level of individual genes, we reasoned that gene network modeling would be a more powerful approach to provide a broader characterization of the biological changes induced by THC treatment in the brain. Therefore, we constructed WGCNA gene coexpression networks for each sex within each brain region (Table S3) and performed trait-module correlation analysis to identify modules (groups of genes highly coexpressed or coregulated) that are significantly correlated with THC treatment and/or cognitive traits impaired by THC $(p<$ 0.05, Table S4). As shown in figure 3A, this analysis identified 29 modules significantly correlated with THC treatment (referred to as "THC-correlated modules") and 12 modules significantly correlated with memory traits from the 6-DOT. Consistent with the DEG analysis, a larger number of THC-correlated modules was observed in female Amy $(n=9)$ and male NAc samples $(n=6)$, while male PFC, DMS and Amy modules showed no correlation with THC treatment or memory traits (Fig. 3A). Social preference was correlated with 9 modules; however, there was no overlap with THC-correlated modules (Table S4, Fig. 3B-C). 

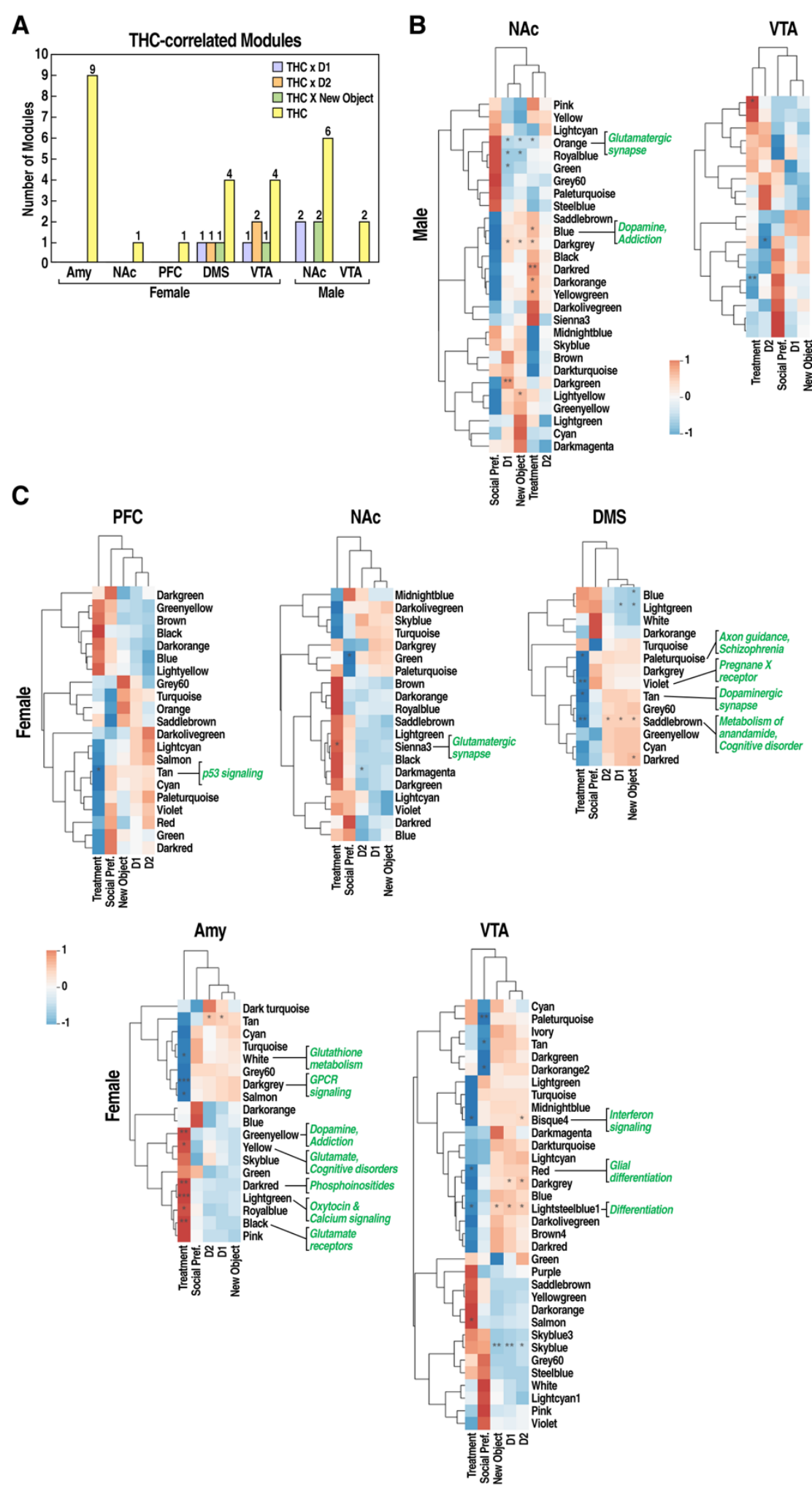

Figure 3:

Coexpression

modules correlated

with chronic THC

administration and

mouse cognitive

phenotypes. (A)

Bar plot of the

number of

coexpression

modules significantly

correlated with $\mathrm{THC}$

treatment and

cognitive

phenotypes $(p<$

0.05). (B-C)

Heatmaps of module

correlation with $\mathrm{THC}$

treatment and

cognitive

phenotypes across

brain regions in

males (B) and

females (C). Color

depicts direction and

strength of correlation. Pathway annotations of selected modules were shown. ${ }^{*} p<0.05 ;{ }^{* *} p<0.01 ;{ }^{* * *} p$

< 0.001. DMS, dorsomedial striatum; Amy, amygdala; NAc, nucleus accumbens; PFC, prefrontal cortex;

VTA, ventral tegmental area; D1, absolute discrimination index 1, D2, relative discrimination index 2. 
We found minimal overlap between the THC-correlated modules and DEGs (Suppl. Fig. 4), indicating that network analysis captures additional information about responses of coordinated gene sets that go beyond changes in individual DEGs.

Pathway enrichment analysis of THC-correlated modules revealed that some pathways were uniquely enriched in specific THC-correlated modules, while others were shared among several modules (Fig. 3B for male modules and Fig. 3C for female modules, Table S5). Among uniquely regulated pathways, we identified glutathione metabolism in female Amy (white), p53 signaling in female PFC (tan), pregnane X receptor pathway in female DMS (violet), glial differentiation in female VTA (red), and phosphonate metabolism in male VTA (cyan). Among the shared pathways, the greenyellow module from female Amy and the blue module from male NAc were enriched for genes implicated in dopaminergic neurotransmission and addiction-related pathways, and were both positively correlated with THC treatment. In addition, the tan module from female DMS was related to dopamine signaling but negatively correlated with THC. Other THC-correlated modules in female Amy (black, yellow) and male NAc (orange) were related to similar pathways, such as regulation of glutamatergic synapse, but displayed positive correlation in females and negative correlation in males with THC.

Further, we identified modules that were simultaneously correlated with THC treatment and memory traits, thus revealing gene networks that were directly associated with cognitive deficits observed in mice following adolescent exposure to THC. Among these modules, saddlebrown (enriched for genes involved in the metabolism of endogenous cannabinoids) in the female DMS, bisque4 (enriched for interferon signaling genes) and lightsteelblue1 (enriched for genes related to differentiation 
processes) in the female VTA were negatively correlated with THC treatment but positively correlated with memory traits. Finally, in the male NAc, darkgrey (no pathways enriched) showed positive correlations with both THC and memory traits but orange (enriched for synaptic transmission genes) showed a negative correlation with both THC and memory traits.

To better understand the regulatory mechanisms underlying changes in transcriptional responses to THC, we identified 104 "hub genes" based on intra-modular connectivity in WGCNA coexpression networks and 146 "key driver genes" based on causal gene regulatory relationships in brain Bayesian networks (Table S6). Among the hub genes of WGCNA coexpression modules that showed correlation with both THC and memory traits, several were involved in the regulation of memory processes. These hub genes included Lynx1 (saddlebrown, Suppl. Fig. 5A) which suppresses memory processes by inhibiting nicotinic receptors-induced neuronal plasticity ${ }^{21,22 ;}$; Cyp46a1 (saddlebrown, Suppl. Fig. 5B) which catalyzes cholesterol degradation and is linked to neuroprotection ${ }^{23}$; Sgp/1 (bisque4, Suppl. Fig. 5B) which is implicated in lipid metabolism and cognitive functions ${ }^{24}$; Gng7 (lightsteelblue1, Suppl. Fig. 5C) which has a role in dopamine receptor stimulation and cognitive abilities ${ }^{25,26}$.

Moreover, among the key driver genes in Bayesian gene regulatory networks, we identified the dopamine receptor Drd1 and the orphan G-protein-coupled receptor Gpr88, which were shared between female Amy greenyellow and male NAc blue modules (Suppl. Fig. 5D). These genes are known regulators of addiction processes $^{27,2829}$, which agrees with the pathway enrichment analysis of Amy greenyellow and male NAc blue modules. 
To identify cell type contributions to coexpression modules, we performed celltype marker enrichment analysis within each THC-correlated module. In female Amy, female NAc and male NAc, modules positively correlated with THC showed enrichment of pyramidal neuron markers, while modules negatively correlated with THC showed enrichment for interneuron markers (Suppl. Fig. 6A). The cell types implicated were consistent with their association with pathways related to dopamine signaling, addiction and glutamatergic synapse. In contrast, 3 out of 4 modules from female VTA were negatively correlated with THC and enriched for markers for astrocytes, oligodendrocytes and microglia (Suppl. Fig. 6B), which was supported by the immune functions revealed by the pathway analysis of the modules (Fig. 3). Lastly, both neuronal and non-neuronal cells contributed to cell type enrichment in female DMS and male VTA THC-correlated modules (Suppl. Fig. 6C).

Taken together, these results indicates that distinct gene coexpression networks are influenced by THC in a sex-, brain region- and cell type-specific manner, and identify new pathways and genes that are potential regulators of the long-term behavioral effects of adolescent exposure to THC, including cognitive impairments and addictive behaviors.

\section{Cross-brain region module-module interactions affected by THC}

Next, we analyzed the correlations between modules within and between brain regions to identify potential intra- or inter-regional molecular circuitries that are disrupted by THC. Thus, we define "THC-interconnected modules" as those significantly correlated 
A Female THC-interconnected modules

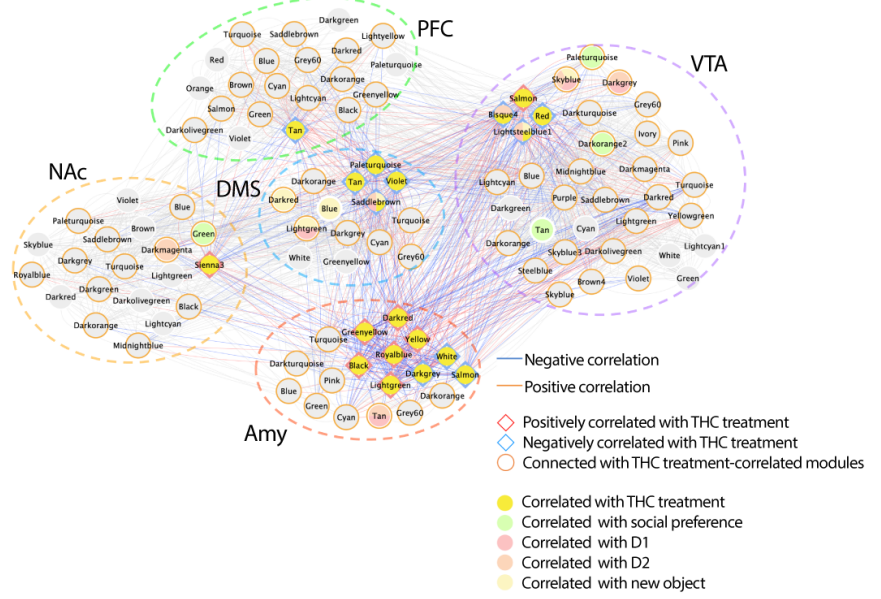

B

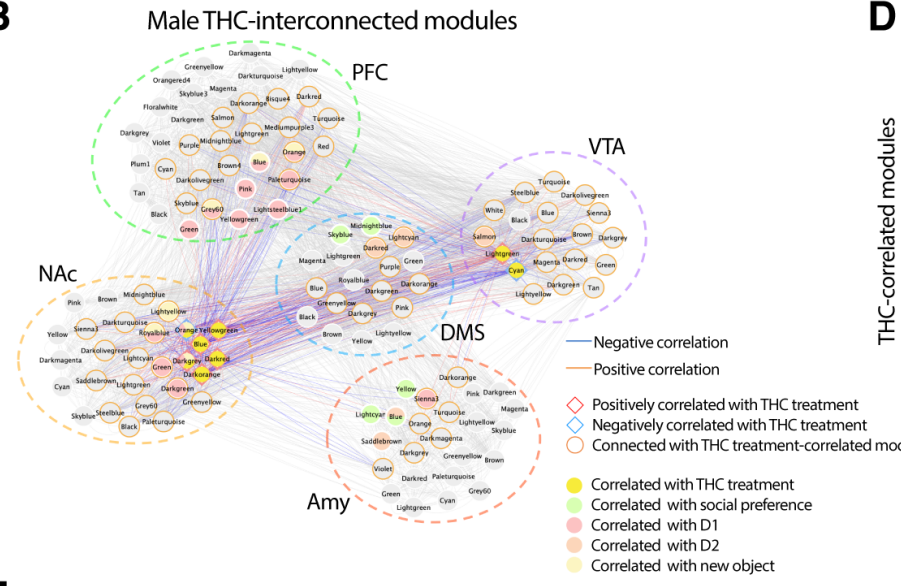

E

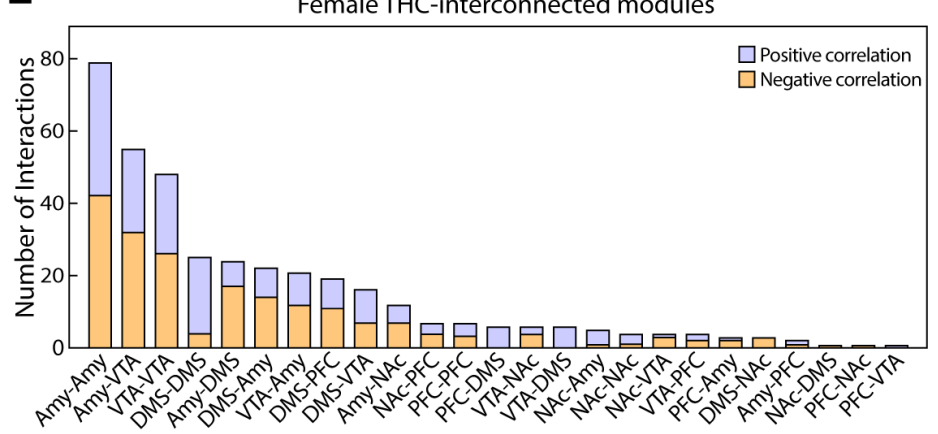

C FemaleTHC-interconnected modules

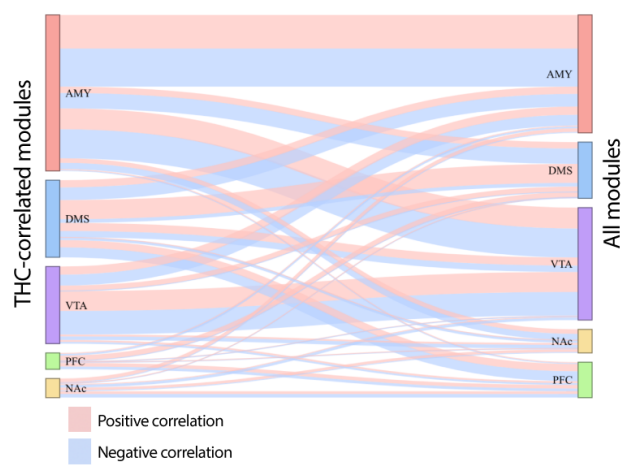

D MaleTHC-interconnected modules

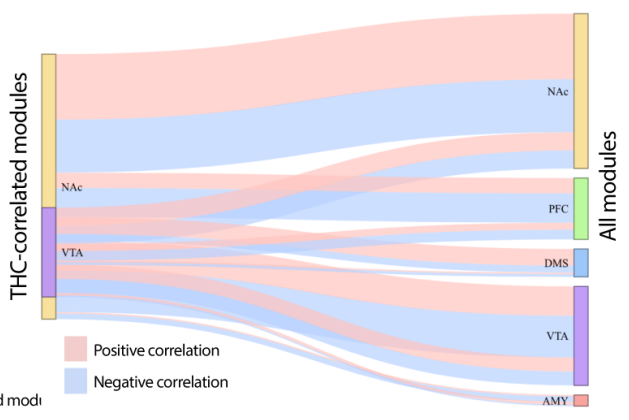

$\mathbf{F}$

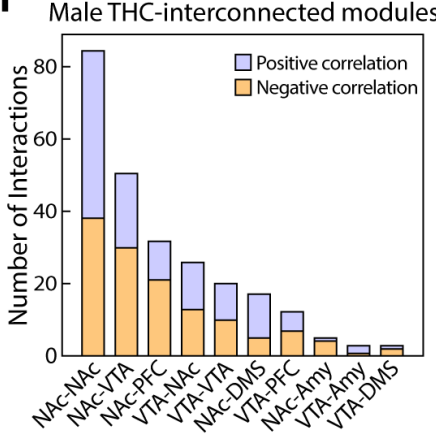

Figure 4: Construction of THC-interconnected module map reveals potential intra- or inter-region molecular circuitries disrupted by THC. (A-B) Visualization of female (A) and male (B) THCinterconnected modules with $|r|>0.5$ and $p<0.05$. Edge and node borderline colors denote positive or negative correlation between pairs of modules. Node fill colors denote module correlation relationships. (C-D) Sankey plots of female (C) and male (D) THC-correlated module interactions. Link colors denote the direction of correlation. (E-F) Bar plots of the number of intermodular interactions in THCinterconnected modules. Color denotes the direction of correlation. DMS, dorsomedial striatum; Amy, amygdala; NAc, nucleus accumbens; PFC, prefrontal cortex; VTA, ventral tegmental area. 
with THC-correlated modules with $|r|>0.5$ and $p<0.05$. We identified numerous THCinterconnected modules in both females (Fig. 4A) and males (Fig. 4B), indicating that additional gene networks in the same region or across brain regions are indirectly influenced by THC. As shown by the Sankey diagrams in figure 4C-D, positive and negative correlations were relatively balanced across brain regions in both sexes. In females, higher levels of connectivity were observed between Amy-Amy modules, followed by Amy-VTA, VTA-VTA, DMS-DMS and Amy-DMS (Fig. 4E). In males, higher levels of connectivity were observed between NAc-NAc, followed by NAc-VTA, NAcPFC, VTA-NAc, and VTA-VTA (Fig. 4F).

Overall, these findings suggest that adolescent exposure to THC leads to long term changes in molecular circuitries across different brain regions in a sex-specific manner.

\section{Associations between coexpression modules altered by THC and human traits related to cannabis use}

Next, we examined the relationship of the coexpression networks altered by THC in mice with human phenotypes related to cannabis use, such as those examined in GWAS for cannabis use disorder (CUD), schizophrenia, memory performance, cognitive aspects of educational attainment, and social interaction. Using Mergeomics (Suppl. Fig. 7A- diagram) ${ }^{19}$, we defined "GWAS-associated modules" as those WGCNA modules enriched in GWAS-associated genes (Table S7). THC-correlated modules overlapped with $11.3 \%$ and $6.1 \%$ GWAS-associated modules in females and males, respectively (Suppl. Fig 7B). However, when we included THC-interconnected modules into the analysis, the overlap increased to $79 \%$ and $53.7 \%$ of GWAS-associated modules in females and males, respectively (Suppl. Fig. 6C). This finding suggests a 
potential role of THC-interconnected modules in THC-induced behavioral abnormalities, despite the fact that they are not directly correlated with THC treatment.

To evaluate the biological significance of GWAS-associated modules that respond to $\mathrm{THC}$, we focused on the functional annotations for gene networks that are significantly enriched for genes from at least 3 GWAS, plus significant correlation with THC treatment in mice (inferred from either from THC-correlated or interconnected modules). As shown in figure $5 A-B$, our analysis not only confirmed known pathways relevant to cognitive processes and psychiatric disorders, such as glutamatergic transmission (female NAc blue, Amy yellow, and male NAc orange, DMS darkred and Amy darkorange), dopaminergic transmission (female NAc darkmagenta and VTA blue), ubiquitin mediated proteolysis (female Amy darkorange), axon guidance (female DMS darkorange/paleturquoise and male VTA blue), and immune function (female VTA darkorange, male NAc royalblue), but also pinpointed the brain region and sex specificity for these pathways. Importantly, our analysis also revealed relatively new pathways and their specific tissue and sex contexts, such as glycosaminoglycan metabolism (female PFC black), inositol metabolism (male DMS pink) and PPARalpha signaling (male DMS greenyellow).

To identify potential regulatory components of the gene networks altered by THC and enriched in GWAS-associated genes, we focused on schizophrenia associated genes given the potential link between schizophrenia vulnerability and adolescent cannabis use ${ }^{30}$, and the availability of both common and rare variants from wellpowered GWAS and whole exome sequencing (WES) studies in humans. Here, we report key driver genes of the female Amy yellow and darkorange modules, which were 

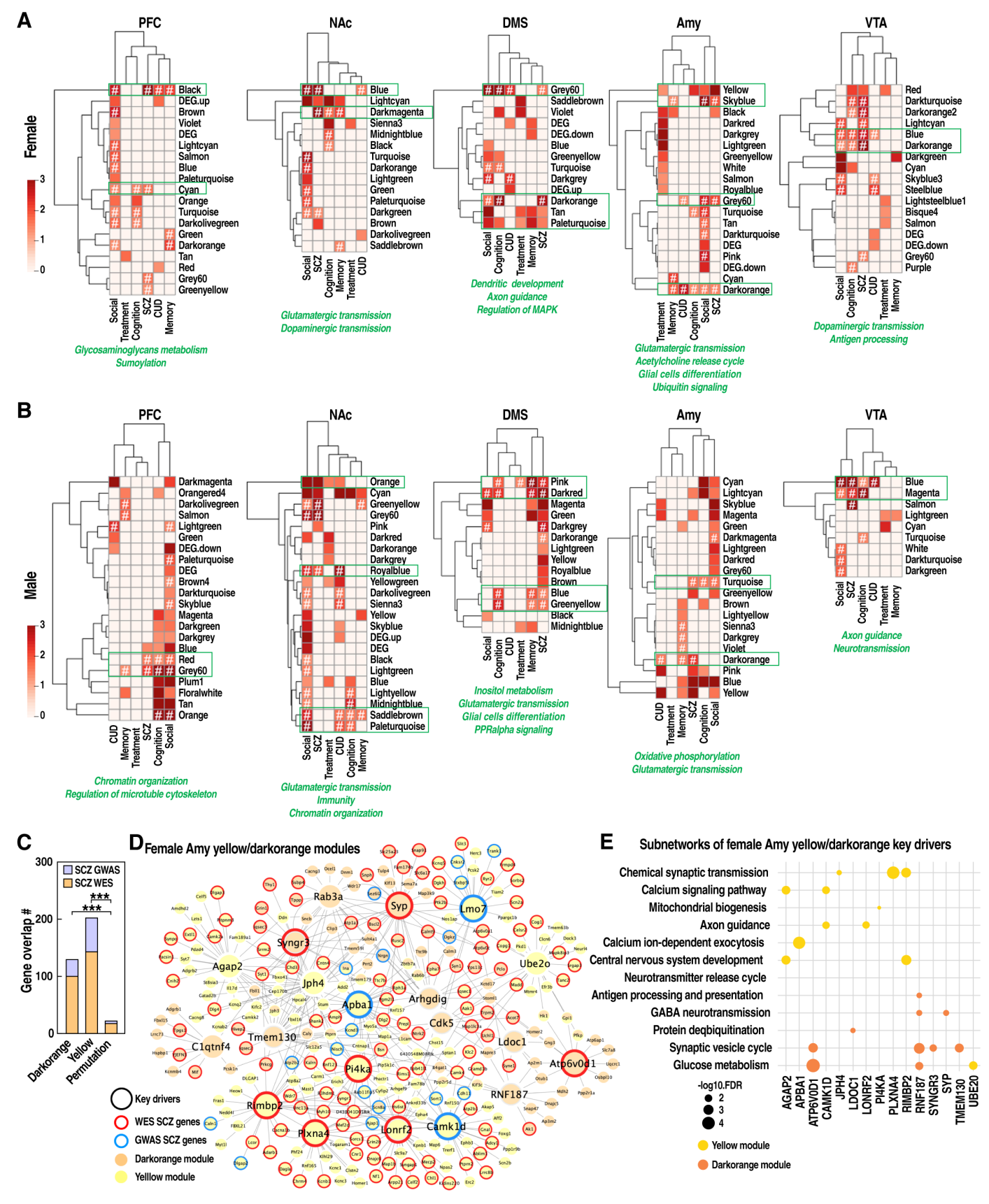

Figure 5: THC-correlated and interconnected modules are associated with human cognitive traits and disorders. (A-B) Heatmaps of the modules that are significantly associated with GWAS traits (cognition, memory, social, CUD, and SCZ) in females (A) and males (B). \# denotes THC-interconnected modules. Color depicts significance in terms of -log(FDR). (C) Bar plot of SCZ variant gene overlap numbers with yellow and darkorange module genes from the female amygdala. ${ }^{* * *} p<0.001$. (D) Visualization of Bayesian network of the female Amy yellow module and darkorange module. Key driver genes are represented by large size nodes. SCZ rare variants discovered by WES are labeled with red borderline and SCZ common variants discovered by GWAS are labeled with blue borderline. Yellow or orange color denotes the origin module of the genes (yellow and darkorange, respectively). (E) Dot plot of subnetwork pathway enrichment. Dot size depicts -log(FDR) value of the enrichment and color depicts yellow or darkorange module.DMS, dorsomedial striatum; Amy, amygdala; NAc, nucleus accumbens; PFC, prefrontal cortex; VTA, ventral tegmental area; CUD, cannabis use disorder; SCZ, schizophrenia; WES, whole-genome sequencing. 
selected because the female Amy transcriptome is more potently altered by THC compared to other brain regions. We found that both yellow and darkorange networks were significantly enriched for common and rare variant genes for schizophrenia as demonstrated by a permutation test (Fig. 6C). As shown in figure 6D, 10 out of 20 key drivers also overlapped with schizophrenia variant genes. The majority of key driver genes encode membrane proteins and are localized at the synapse and vesicles. Besides, several key driver genes are implicated in brain development, including Plxna4, Syngr3, Agap2, Camk1d and Atp6v0d1.

Subnetwork pathway analysis revealed that 10 out of the 20 key driver genes (Jph4, Plxna4, Rimbp2, Rnf187, Syp, Syngr3, Tmem130, Agap2, Apba1 and Camk1d) orchestrate tissue-specific subnetworks of genes implicated in neurotransmission, including chemical synaptic transmission, neurotransmitter release cycle, GABA transmission, synaptic vesicle cycle, and calcium signaling. 5 out of the 20 key drivers (Agap2, Rimbp2, Tmem130, Camk1d, Lonrf2) regulate central nervous system development related subnetworks. Three key drivers (Atp6vod1, Rnf187, and Ube20) regulate glucose metabolism subnetworks. We also identified key drivers (Pi4ka, Rnf187, Ldoc1) regulating mitochondrial biogenesis, antigen processing and protein deubiquitination subnetworks.

Taken together, this integrative analysis supports a strong mechanistic connection between genes and pathways altered by THC and those associated with schizophrenia vulnerability. 


\section{Discussion}

Our work provides the first comprehensive, tissue- and sex-specific view of molecular processes perturbed by adolescent THC treatment in mice. Our findings suggest that females are more sensitive than males to the long-term detrimental effects of THC on recognition memory, which coincides with larger transcriptional responses in the female brain, particularly the Amy region. The gene network analysis identified potential regulators and biological pathways altered by $\mathrm{THC}$, including cognitive and addiction processes. Lastly, by intersecting our mouse transcriptomic data with human GWAS of neuropsychiatric disorders and cognitive behavioral traits, we found a connection between gene networks affected by THC and human traits and disorders known to be influenced by cannabis use, such as schizophrenia and CUD.

In line with previous reports in rodent models ${ }^{16,31-37}$, our behavioral analysis demonstrated that adolescent exposure to THC in mice led to long-term impairments in object recognition memory and social interaction, but not in anxiety-like behaviors. Our study also showed sex differences in the effects of THC on recognition memory, which was was impaired more in female compared to male mice. While the influence of sex on the effects of THC on memory has not been evaluated in animal models, female rats have been reported to be more susceptible than males to the effects of THC on locomotor activity, nociception and reward processes ${ }^{38-42}$. Importantly, the greater sensitivity of females to the harmful effects of THC on spatial memory is evident also in humans ${ }^{43-47}$. Overall, our behavioral data confirms the harmful effect of THC on cognitive functions and highlights the importance of addressing sex differences when studying the long-term impact of cannabis use. 
Sex differences in gene expression patterns may elucidate the mechanisms underlying sexual dimorphism in behavioral phenotypes in response to drugs ${ }^{48}$. In agreement with this hypothesis, our study demonstrated that adolescent exposure to THC caused sex-specific gene expression changes in the brain. When we conducted a statistical analysis that explicitly tested for sex differences in DEGs, we found significant sex $x$ treatment interaction in Amy, NAc and DMS. The sex-specific gene expression differences could also influence other mechanisms known to play an important role in determining THC effects on females and males, including sex-specific effects of hormonal changes on the endocannabinoid system during adolescence ${ }^{49}$, differential density of cannabinoid receptors in the brain ${ }^{50}$, and pharmacokinetic factors ${ }^{51}$.

Taken together, these results suggest that female mice with a history of adolescent exposure to THC exhibit more profound cognitive behavioral deficits and larger transcriptional alterations than males.

It is unknown what particular brain region drives the behavioral abnormalities induced by adolescent exposure to THC. Our work that, for the first time, simultaneously investigates 5 brain regions, suggests an extensive brain region specificity in the gene signatures and networks altered by THC. The analysis of sexually dimorphic DEGs and THC-correlated modules revealed that female Amy and male NAc may be sites of particular importance for the persistent changes induced by adolescent exposure to THC. In line with these findings, brain morphological studies of human cannabis users have shown that marijuana use may be associated with the disruption of neural organization of the Amy and $\mathrm{NAc}^{52}$. Moreover, previous studies have documented that female teenagers who use marijuana are more susceptible than males to structural 
abnormalities of the Amy, which were correlated with worse internalizing symptoms ${ }^{53}$. Consistent with these observations, animal studies also reported perturbation of synaptic transmission in the Amy and NAc following administration of exogenous cannabinoids $^{54-56}$. However, previous analysis of transcriptomic changes induced by adolescent exposure to THC have focused only on PFC of male rats ${ }^{10}$. Miller et al reported that adolescent exposure to THC was associated with long-term gene expression changes in the PFC, which were related to cytoskeleton and chromatin regulation. While we did not identify any DEGs or THC-correlated modules in the male PFC in mice, we found 2 THC-interconnected modules in the male PFC, which were linked to the same biological pathways. Between-species differences may explain the discrepancy.

Our multiple brain region studies also allowed us to uniquely infer network connections within and between brain regions. The THC-interconnected modules are likely indirectly influenced by THC, as they are inferred from the module-module interaction network. We speculate that the inter-region interaction network could predict how THC directly influences one brain region which then cascades down to other brain regions. Specifically, our analysis predicts that adolescent exposure to THC alters neural circuits that connect Amy with VTA and DMS in females, and neural circuits that connect NAc with VTA and PFC in males. Future experiments perturbing the THC modules using animal models will be necessary to validate these predictions.

A major strength of this study is that we can directly correlate the transcriptional responses to $\mathrm{THC}$ with the cognitive traits altered by $\mathrm{THC}$ in the same mice. The traitmodule correlation analysis suggested that the disruption of endogenous cannabinoid 
signaling in the DMS and inflammatory pathways in the VTA may drive the deficits in recognition memory observed in female mice. We also showed that neuronal cell types in the DMS and glial cells (astrocytes and microglia) in the VTA may contribute to the transcriptional response to THC underlying memory deficits. In line with these results, it is well known that the endocannabinoid system plays an essential role in learning and memory processes, with the engagement of the dorsal striatum specifically in encoding habit-related memories ${ }^{57-59}$. Moreover, repetitive exposure to synthetic cannabinoids led to inflammatory phenotypes including astrogliosis in the VTA ${ }^{60}$.

The frequent use of cannabis during adolescence has been linked to deleterious long-term consequences in humans, including an elevated likelihood of earlier onset of schizophrenia, cannabis use disorders and addiction ${ }^{30,61-68}$. We found coexpression modules that both respond to THC treatment and are enriched in genes relevant to human cognitive traits, schizophrenia, and CUD. These modules are involved in pathways already reported to be implicated in the development of psychiatric disorders, such as synaptic transmission, dopaminergic transmission, axon guidance, MAPK regulation, chromatin organization and immune functions ${ }^{61-67}$. We have also identified novel pathways that link chronic adolescent THC exposure to schizophrenia, such as sumoylation and glycosaminoglycan metabolism in females and inositol metabolism in males. This analysis also revealed that three key driver genes (Atp60vd1, Rnf187, Ube20) regulate glucose metabolism, suggesting a novel link between THC use and schizophrenia, which is also supported by evidence in human studies ${ }^{69}$. 
Taken together, our results corroborate previous findings on the correlation of cannabis use with negative mental outcomes, and pinpoint potential coexpression modules, pathways and genes driving pathological mechanisms.

Our results should be considered in light of certain limitations. First, we focus on correlating transcriptomic changes that occur during adulthood with behaviors measured at the same time. This approach has the advantage of capturing persistent changes associated with a history of adolescent exposure to THC; however, it cannot directly assess the earlier transcriptional and behavioral changes during the adolescent period, which we hypothesize to be particularly vulnerable to THC. The second limitation of our study is that we focus on cognitive behaviors, such as recognition memory and social interaction. However, other behaviors are likely to be influenced by THC, including addiction-like phenotypes. It is important to note, however, that addictive properties of THC are not well modeled in mice. Thirdly, our study is limited to 5 brain regions and other additional gene regulatory networks can be missed. Thus, it would be important to expand this study to other brain regions, such as the hippocampus given its critical role in recognition memory. Lastly, our studies open numerous new hypotheses that warrant future experimental validation.

In summary, our study is the first to unveil the potential molecular mechanisms underpinning the persistent behavioral alterations linked to adolescent cannabis use in a sex- and brain region-specific manner. 

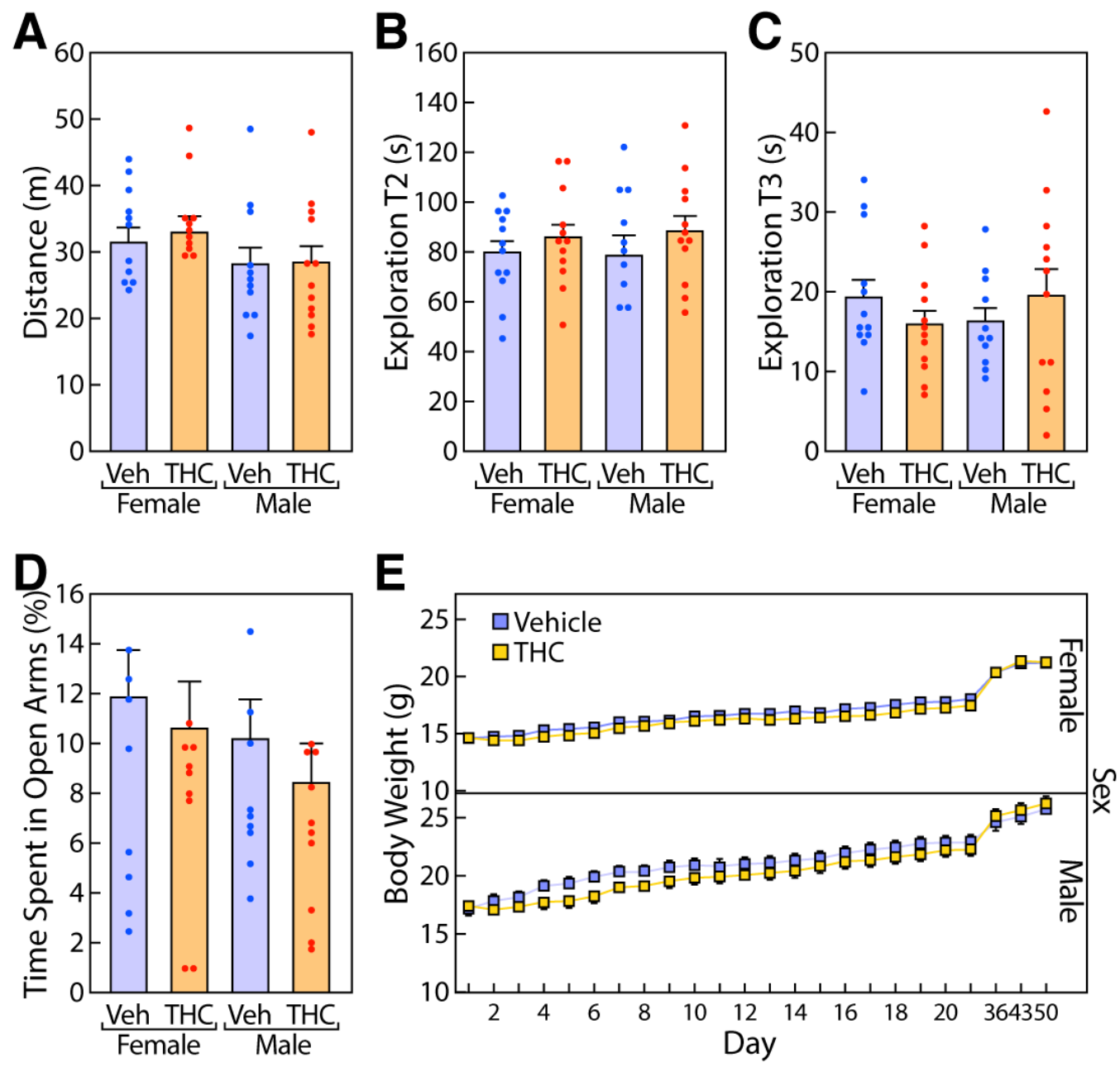

Supplementary Figure 1: Behavioral characterization of female and male mice following adolescent exposure to THC. (A) Locomotor activity is expressed as mean distance traveled $(\mathrm{m}) \pm \mathrm{SEM}$. Exploration $(\mathrm{s})$ in familiarization phase $\mathrm{T} 2(\mathrm{~B})$ and test phase T3 (C) are expressed as mean \pm SEM. (D) Body weight $(\mathrm{g})$ is expressed as mean \pm SEM for the THC administration course (day 1 to 21 ), and for day 36,43 , and 60 when the three behavioral assays started. All effect and interaction $p$ values $>0.1$. 
A

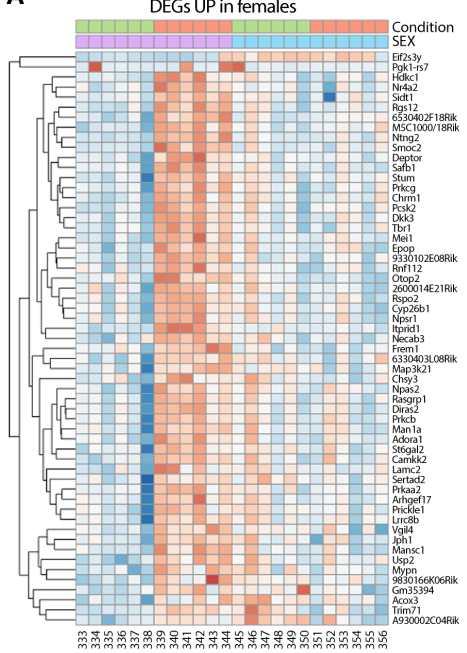

Amy

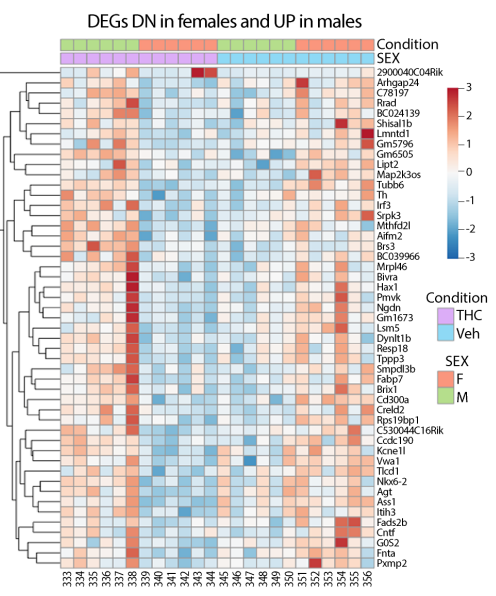

B

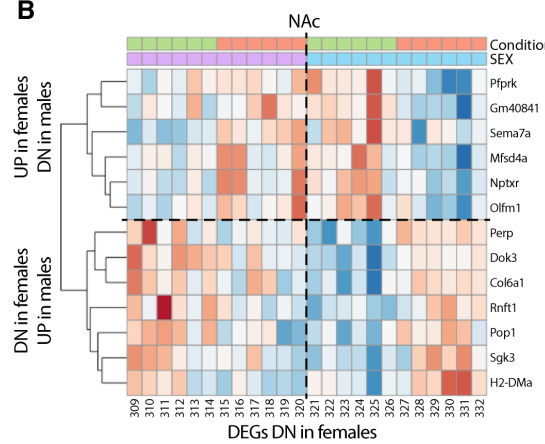

C DMS

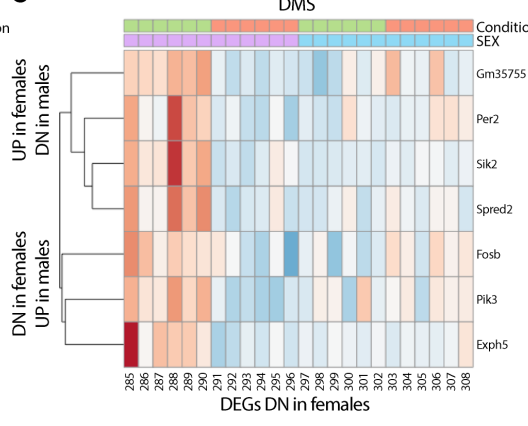

Supplementary Figure 2: Adolescent THC administration induced sex-specific

transcriptional changes. Heatmaps of treatment by sex interaction DEGs in Amy (A), NAc (B), and DMS (C). DMS, dorsomedial striatum; Amy, amygdala; NAc, nucleus accumbens; Veh, vehicle; DEG, differential expression gene; UP, upregulated; DN, downregulated. 
A
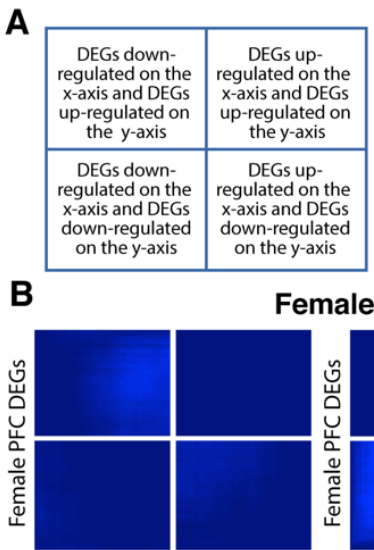

Female Amy DEGs

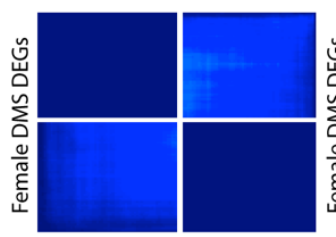

Female PFC DEGs

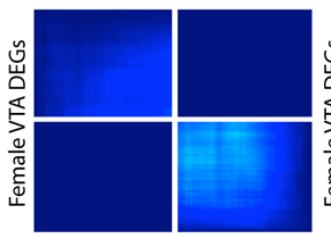

Female PFC DEGs

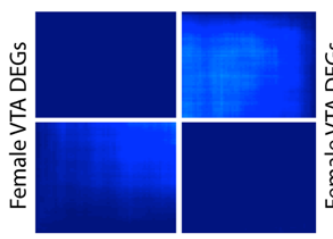

Female DMS DEGs

D

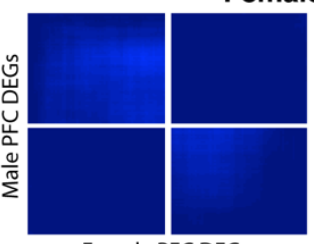

Female PFC DEGs

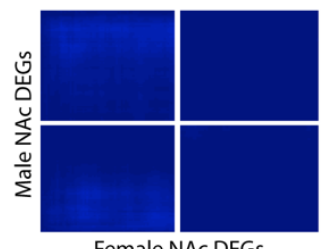

Female NAc DEGs

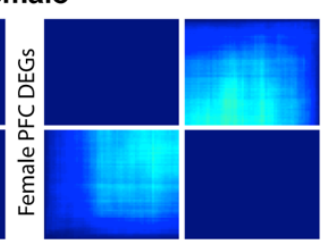

Female NAc DEGs

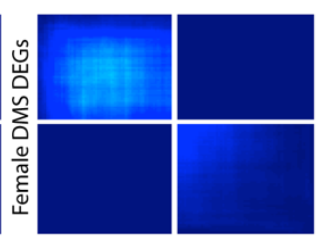

Female NAc DEGs

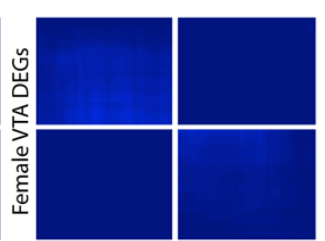

Female NAc DEGs

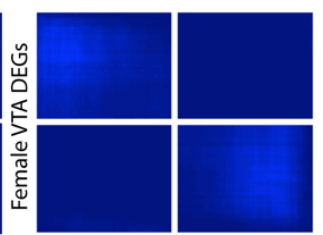

Female Amy DEGs

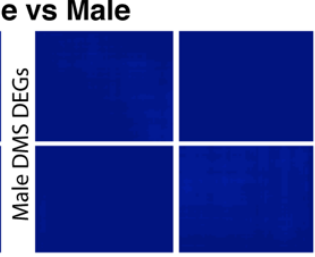

Female DMS DEGs

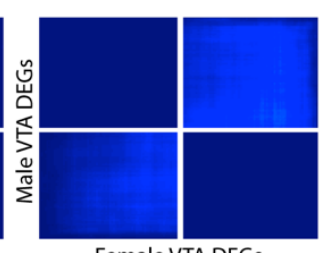

Female VTA DEGs

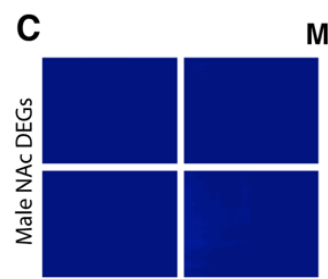

Male Amy DEGs

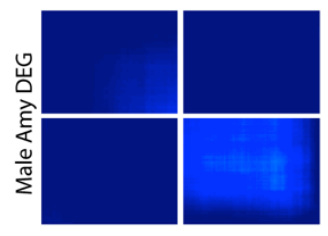

Male PFC DEG

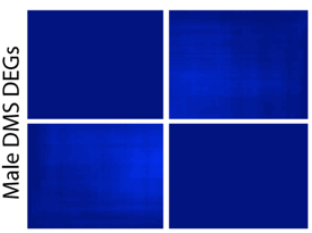

Male PFC DEGs

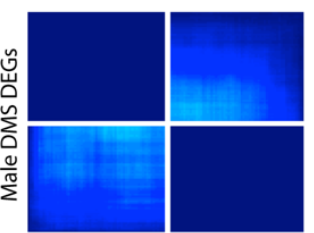

Male NAc DEGs

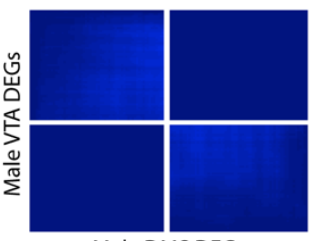

Male DMS DEGs

E
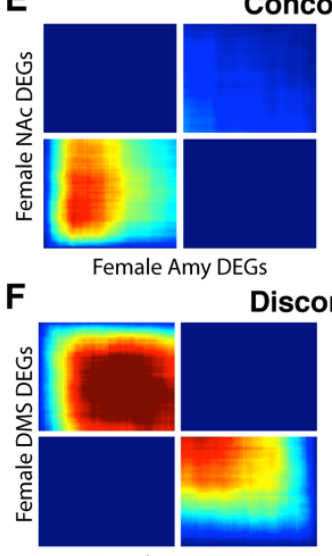

Female Amy DEGs

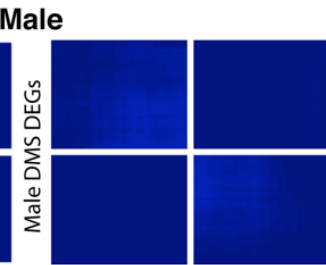

Male Amy DEGs

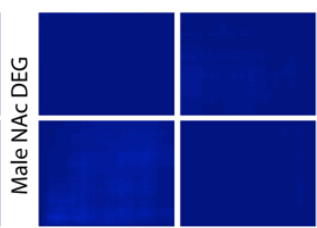

Male PFC DEG

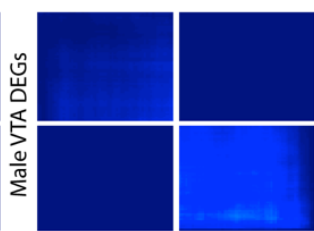

Male PFC DEGs

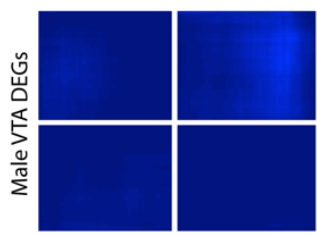

Male NAc DEGs

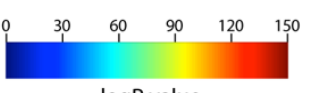

- $\log \mathrm{P}$ value

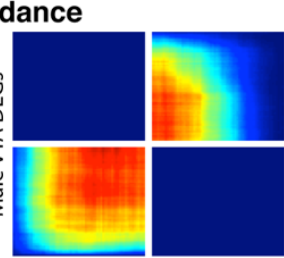

Male Amy DEGs

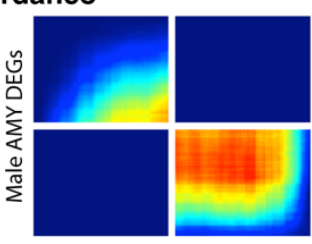

Female Amy DEGs

\section{Supplementary}

Figure 3. Minimal transcriptional

\section{overlap was}

detected in most comparisons across sex and brain regions.

(A)Schematic of RRHO heatmap interpretation. (BD) $\mathrm{RRHO}$ heatmaps show minimal overlap in transcription between pairs of brain regions within females (B), males (C), and between sexes (D). (E-F) RRHO heatmaps show concordance (E) and discordance $(F)$ in transcription between pairs of brain regions. We define concordant as positive correlation and discordant as negative correlation in DEG overlap patterns.

Amy, amygdala; DMS, dorsomedial striatum; VTA, ventral tegmental area; PFC, prefrontal cortex; NAc, nucleus accumbens; DEG, differential expression gene. 

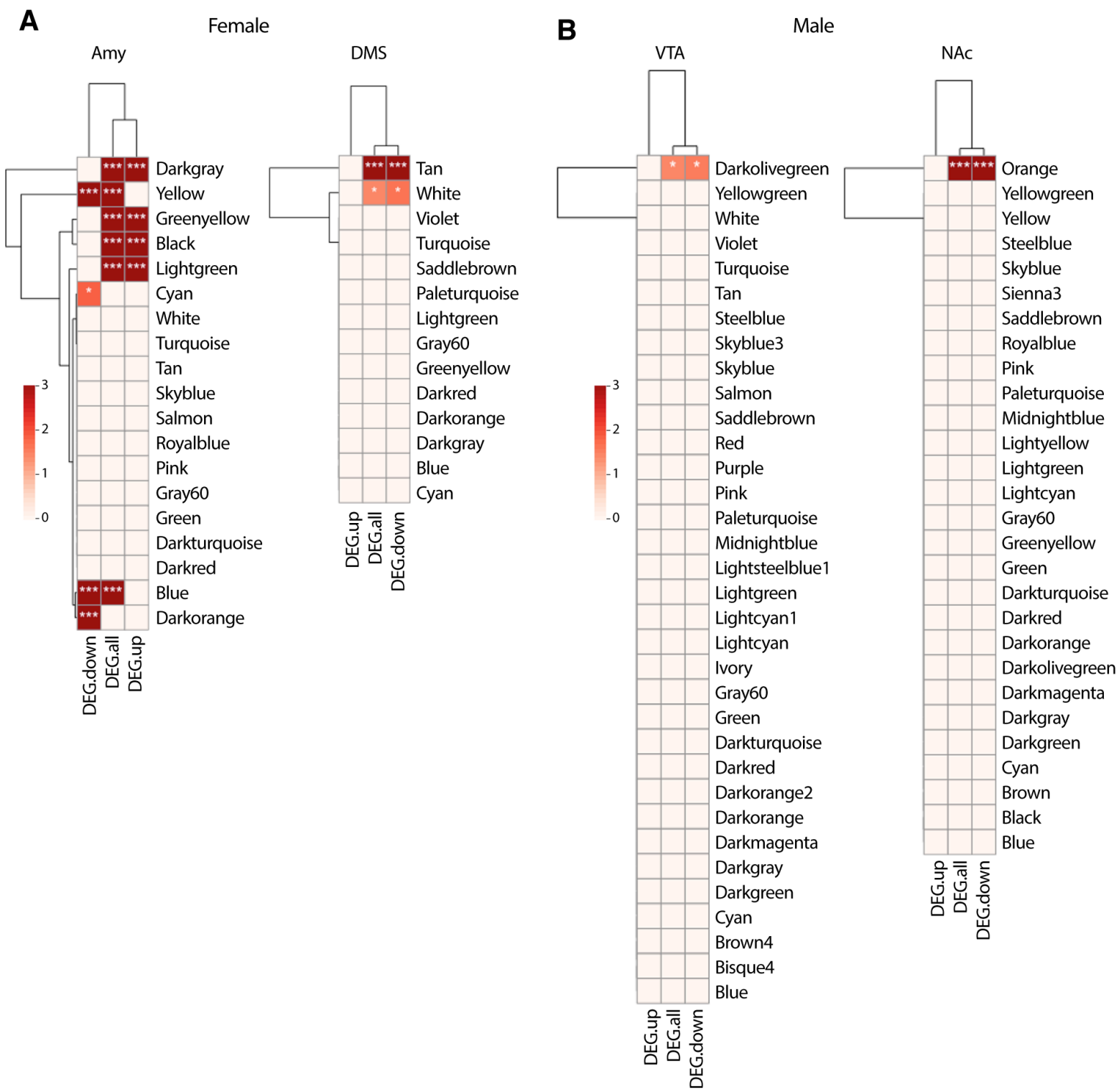

\section{Supplementary Figure 4: DEG enrichment in coexpression modules was detected in a few brain} regions. (A-B) Heatmaps of DEG enrichment in brain regions. Only female Amy, DMS and male VTA, and NAc showed significant enrichment in DEGs. Color depicts - $\log 10 \mathrm{P}$ value of the enrichment and only significant enrichments were illustrated. ${ }^{*} p<0.05$; ${ }^{* *} p<0.01$; ${ }^{* *} p<0.001$. DMS, dorsomedial striatum; Amy, amygdala; NAc, nucleus accumbens; VTA, ventral tegmental area; DEG.all, all of the differential expression genes of a certain brain region; DEG.uo, upregulated differential expression genes of a certain brain region; DEG.down, downregulated differential expression genes of a certain brain region. 
A

Female DMS saddlebrown

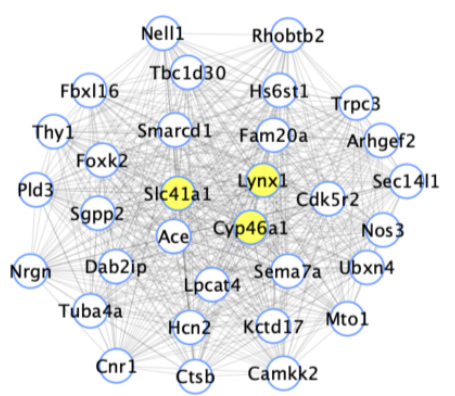

C

Female VTA lightsteelblue1
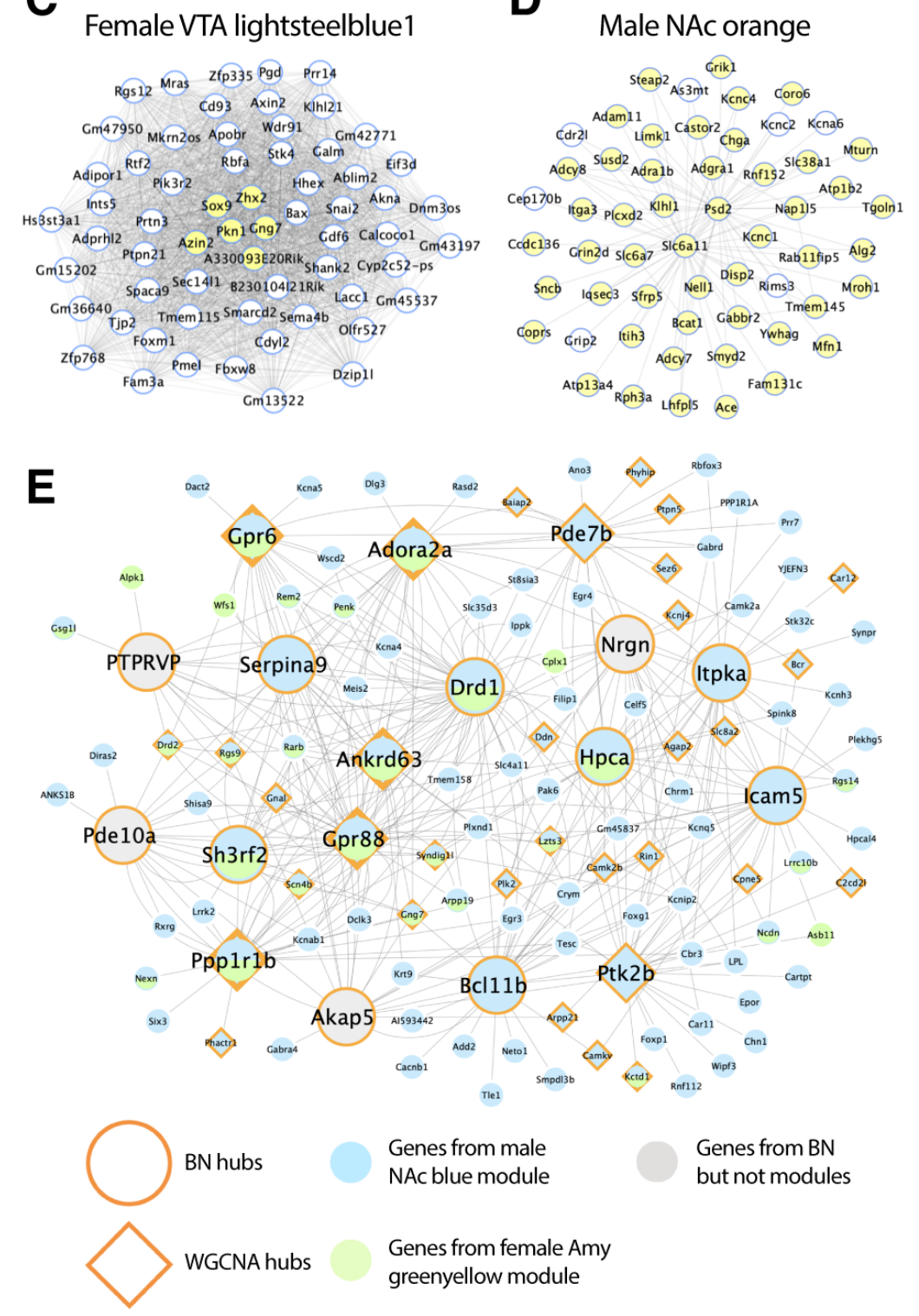

Supplementary Figure 5: Hub genes and key driver genes were identified in THC-correlated coexpression modules. (A-

D) Visualization of coexpression modules correlated with both $\mathrm{THC}$ administration and recognition memory traits. The edges denote positive correlations between pairs of genes by the definition of signed coexpression networks. Hub genes are highlighted in yellow. Only the top 100 edges based on topological overlap weight were visualized due to the large size of the male NAc orange module. (E) Visualization of Bayesian network of the male NAc blue module and female amygdala greenyellow module. Hub genes and key driver genes are labeled with orange borderlines. Key driver genes are illustrated by enlarged circles, overlaps between key driver genes and hub genes are illustrated by enlarged diamonds, and hub genes are illustrated by diamonds. Blue or green color denotes the origin module of the genes and grey color denotes genes from the BN but not in the WGCNA modules. DMS, dorsomedial striatum; Amy, amygdala; NAc, nucleus accumbens; VTA, ventral tegmental area; BN, Bayesian network. 

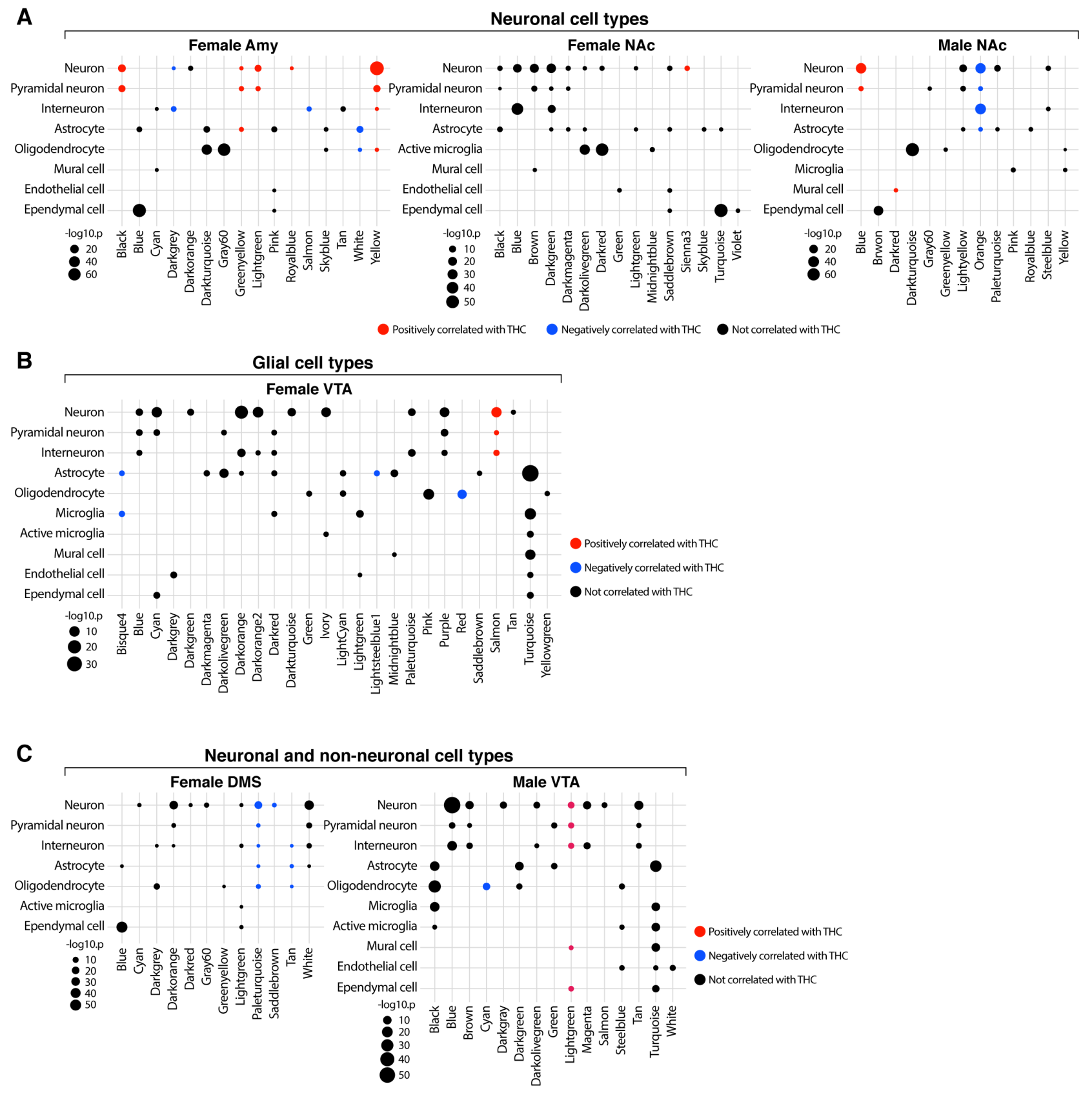

\section{Supplementary Figure 6: THC-correlated coexpression modules exhibit cell-type marker}

enrichments. (A) Dot plots of brain regions with THC-correlated modules enriched in neuronal cell markers. (B) Dot plots of brain regions with THC-correlated modules enriched in glial cell markers. (C) Dot plots of brain regions with THC-correlated modules enriched in both neuronal and non-neuronal cell markers. Dot color depicts the direction of correlation with chronic THC administration. Dot size depicts the significance of the enrichment pattern. Modules not shown are not enriched in cell-type markers. DMS, dorsomedial striatum; Amy, amygdala; NAc, nucleus accumbens; PFC, prefrontal cortex; VTA, ventral tegmental area. 
A

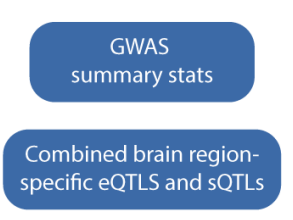

Gene-locus

distance map
Mergeomics pipeline

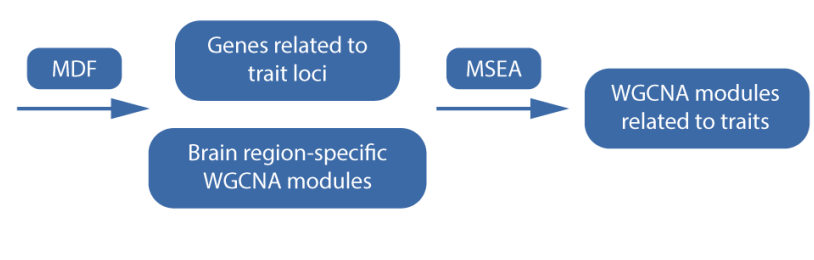

B

B THC-correlated GWAS-associated

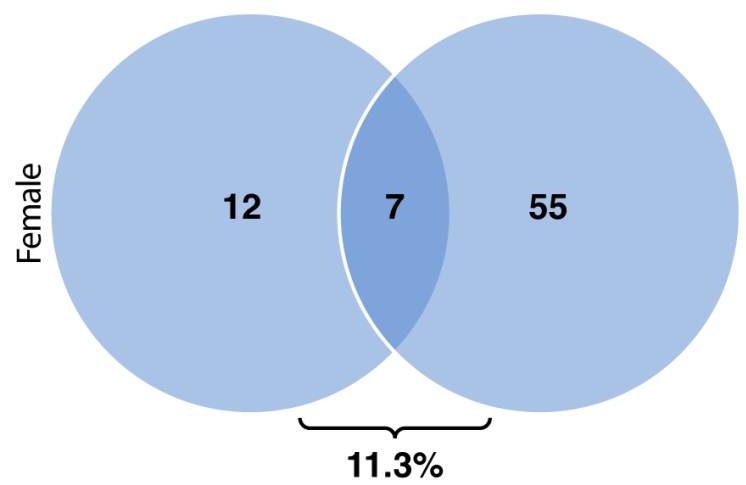

C

C THC-correlated \& interconnected GWAS-associated

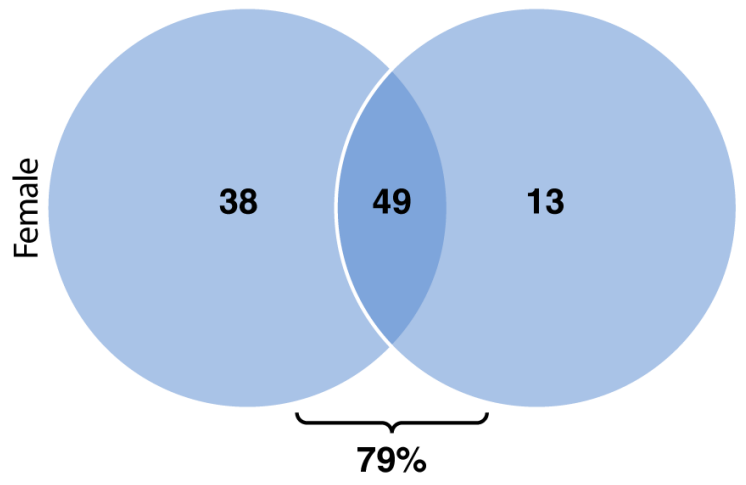

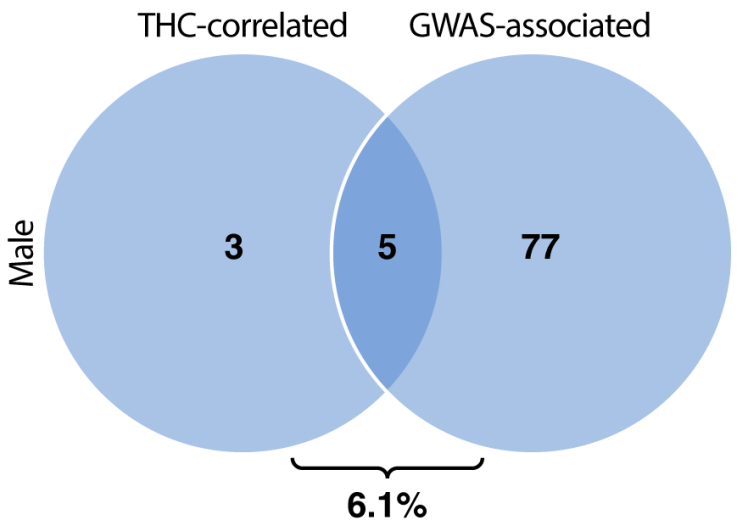

THC-correlated \& interconnected GWAS-associated

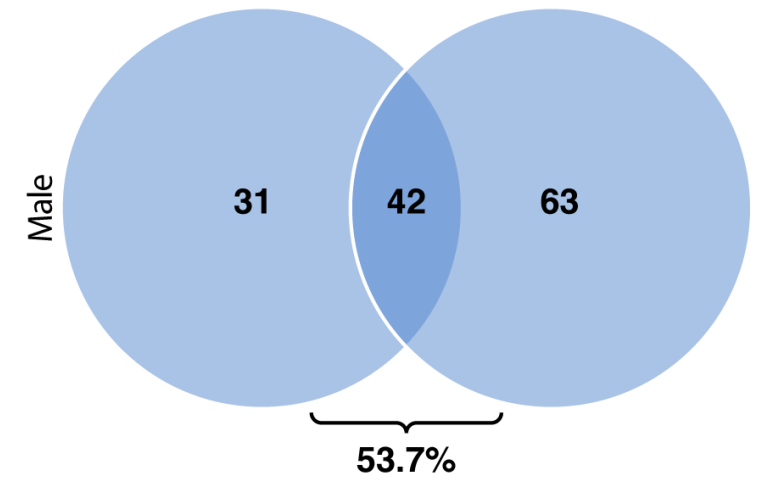

Supplementary Figure 7: Identification of GWAS-associated modules using Mergeomics. (A) Schematic of Mergeomics pipeline. (B) Percentage of overlap in GWAS-associated modules with THCcorrelated modules. (C) Percentage of overlap in GWAS-associated modules with THC-correlated and interconnected modules. Percentage of overlap is calculated as the number of overlapped modules divided by the total number of GWAS-associated modules. MDF, marker dependency filtering; MSEA, marker set enrichment analysis; QTL, quantitative trait loci. 


\section{Acknowledgments}

This work was supported by the National Institute on Drug Use, USA [DP1DA042232, U01DA051972, and U01DA050239 to FT]. XY is supported by the National Center for Advancing Translational Sciences UCLA CTSI Grant UL1TR001881. This publication includes data generated at the UC San Diego IGM Genomics Center utilizing an Illumina NovaSeq 6000 that was purchased with funding from a National Institutes of Health SIG grant (\#S10 OD026929)

FT designed and coordinated the study. XY designed the overall bioinformatics analysis flow. YZ conducted bioinformatic analysis and data interpretation with inputs from FT and $\mathrm{XY}$. Al conducted the behavioral experiments and collected brain samples. FT conducted the statistical analysis of behavioral experiments. PMP processed samples for RNA-sequencing. $\mathrm{HL}$ performed raw data processing. $\mathrm{YZ}, \mathrm{XY}$ and $\mathrm{FT}$ drafted the manuscript. We would like to acknowledge $\mathrm{H}$. Taylor for technical assistance and $\mathrm{J}$. Hightower for figure preparation.

\section{Disclosures}

The authors have no conflicts of interest to disclose. 


\section{References}

1. Curran, H. V. et al. Keep off the grass? Cannabis, cognition and addiction. Nat. Rev.

Neurosci. 17, 293-306 (2016).

2. Di Forti, M. et al. The contribution of cannabis use to variation in the incidence of psychotic disorder across Europe (EU-GEI): a multicentre case-control study. Lancet Psychiatry 6, 427-436 (2019).

3. Di Forti, M. et al. Proportion of patients in south London with first-episode psychosis attributable to use of high potency cannabis: a case-control study. Lancet Psychiatry 2, 233-238 (2015).

4. Silins, E. et al. Young adult sequelae of adolescent cannabis use: an integrative analysis. Lancet Psychiatry 1, 286-293 (2014).

5. Volkow, N. D. Effects of Cannabis Use on Human Behavior-Reply. JAMA Psychiatry 73, 996 (2016).

6. Sturman, D. A. \& Moghaddam, B. The neurobiology of adolescence: changes in brain architecture, functional dynamics, and behavioral tendencies. Neurosci. Biobehav. Rev. 35, 1704-1712 (2011).

7. Maccarrone, M., Guzman, M., Mackie, K., Doherty, P. \& Harkany, T. Programming of neural cells by (endo)cannabinoids: from physiological rules to emerging therapies. Nat. Rev. Neurosci. 15, 786-801 (2014).

8. Hurd, Y. L., Michaelides, M., Miller, M. L. \& Jutras-Aswad, D. Trajectory of adolescent cannabis use on addiction vulnerability. Neuropharmacology 76 Pt B, 416-424 (2014).

9. Hamilton, P. J. \& Nestler, E. J. Epigenetics and addiction. Curr. Opin. Neurobiol. 59, 128136 (2019).

10. Miller, M. L. et al. Adolescent exposure to Delta(9)-tetrahydrocannabinol alters the transcriptional trajectory and dendritic architecture of prefrontal pyramidal neurons. Mol. Psychiatry 24, 588-600 (2019). 
11. Caballero, A., Granberg, R. \& Tseng, K. Y. Mechanisms contributing to prefrontal cortex maturation during adolescence. Neurosci. Biobehav. Rev. 70, 4-12 (2016).

12. Carlezon, W. A., Jr \& Thomas, M. J. Biological substrates of reward and aversion: a nucleus accumbens activity hypothesis. Neuropharmacology 56 Suppl 1, 122-132 (2009).

13. Lipton, D. M., Gonzales, B. J. \& Citri, A. Dorsal Striatal Circuits for Habits, Compulsions and Addictions. Frontiers in Systems Neuroscience vol. 13 (2019).

14. Janak, P. H. \& Tye, K. M. From circuits to behaviour in the amygdala. Nature 517, 284-292 (2015).

15. Bariselli, S., Glangetas, C., Tzanoulinou, S. \& Bellone, C. Ventral tegmental area subcircuits process rewarding and aversive experiences. J. Neurochem. 139, 1071-1080 (2016).

16. Iemolo, A. et al. Reelin deficiency contributes to long-term behavioral abnormalities induced by chronic adolescent exposure to Delta9-tetrahydrocannabinol in mice.

Neuropharmacology 187, 108495 (2021).

17. Zhou, X., Barkley-Levenson, A. M., Montilla-Perez, P., Telese, F. \& Palmer, A. A. Functional validation of a finding from a mouse genome-wide association study shows that Azi2 influences the acute locomotor simulant response to methamphetamine. Genes Brain Behav. 20, e12760 (2021).

18. Shu, L. et al. Shared genetic regulatory networks for cardiovascular disease and type 2 diabetes in multiple populations of diverse ethnicities in the United States. PLoS Genet. 13, e1007040 (2017).

19. Shu, L. et al. Mergeomics: multidimensional data integration to identify pathogenic perturbations to biological systems. BMC Genomics 17, 874 (2016).

20. Sannino, S. et al. Role of the dorsal hippocampus in object memory load. Learn. Mem. 19, 211-218 (2012).

21. Parker, R. L. et al. Deletion of lynx1 reduces the function of $\alpha 6^{*}$ nicotinic receptors. PLoS 
One 12, e0188715 (2017).

22. Miwa, J. M. et al. The prototoxin lynx1 acts on nicotinic acetylcholine receptors to balance neuronal activity and survival in vivo. Neuron 51, 587-600 (2006).

23. Boussicault, L. et al. CYP46A1, the rate-limiting enzyme for cholesterol degradation, is neuroprotective in Huntington's disease. Brain 139, 953-970 (2016).

24. Mitroi, D. N. et al. SGPL1 (sphingosine phosphate lyase 1) modulates neuronal autophagy via phosphatidylethanolamine production. Autophagy 13, 885-899 (2017).

25. Ruano, D. et al. Functional gene group analysis reveals a role of synaptic heterotrimeric $\mathrm{G}$ proteins in cognitive ability. Am. J. Hum. Genet. 86, 113-125 (2010).

26. Schwindinger, W. F. et al. Loss of G protein $y 7$ alters behavior and reduces striatal aolf level and cAMP production. J. Biol. Chem. 278, 6575-6579 (2003).

27. Lobo, M. K. et al. Cell Type-Specific Loss of BDNF Signaling Mimics Optogenetic Control of Cocaine Reward. Science (2010).

28. Wise, R. A. Dopamine, learning and motivation. Nat. Rev. Neurosci. 5, 483-494 (2004).

29. Jin, C. et al. Discovery of a Potent, Selective, and Brain-Penetrant Small Molecule that Activates the Orphan Receptor GPR88 and Reduces Alcohol Intake. J. Med. Chem. 61, 6748-6758 (2018).

30. Volkow, N. D., Baler, R. D., Compton, W. M. \& Weiss, S. R. B. Adverse health effects of marijuana use. N. Engl. J. Med. 370, 2219-2227 (2014).

31. Sabran-Cohen, T., Bright, U., Mizrachi Zer-Aviv, T. \& Akirav, I. Rapamycin prevents the long-term impairing effects of adolescence $\Delta$-9-tetrahydrocannabinol on memory and plasticity in male rats. Eur. J. Neurosci. 54, 6104-6122 (2021).

32. Jouroukhin, Y. et al. Adolescent $\Delta 9$-Tetrahydrocannabinol Exposure and Astrocyte-Specific Genetic Vulnerability Converge on Nuclear Factor-kB-Cyclooxygenase-2 Signaling to Impair Memory in Adulthood. Biol. Psychiatry 85, 891-903 (2019).

33. Saravia, R. et al. Concomitant THC and stress adolescent exposure induces impaired fear 
extinction and related neurobiological changes in adulthood. Neuropharmacology 144, 345-357 (2019).

34. Murphy, M. et al. Chronic Adolescent $\Delta 9$-Tetrahydrocannabinol Treatment of Male Mice Leads to Long-Term Cognitive and Behavioral Dysfunction, Which Are Prevented by Concurrent Cannabidiol Treatment. Cannabis Cannabinoid Res 2, 235-246 (2017).

35. Zamberletti, E., Gabaglio, M., Prini, P., Rubino, T. \& Parolaro, D. Cortical neuroinflammation contributes to long-term cognitive dysfunctions following adolescent delta-9-tetrahydrocannabinol treatment in female rats. Eur. Neuropsychopharmacol. 25, 2404-2415 (2015).

36. Swartzwelder, N. A. et al. Effects of ethanol, $\Delta(9)$-tetrahydrocannabinol, or their combination on object recognition memory and object preference in adolescent and adult male rats. Neurosci. Lett. 527, 11-15 (2012).

37. O'Tuathaigh, C. M. P. et al. Chronic adolescent exposure to $\Delta$-9-tetrahydrocannabinol in COMT mutant mice: impact on psychosis-related and other phenotypes.

Neuropsychopharmacology 35, 2262-2273 (2010).

38. Fattore, L. et al. Cannabinoid self-administration in rats: sex differences and the influence of ovarian function. Br. J. Pharmacol. 152, 795-804 (2007).

39. Craft, R. M., Marusich, J. A. \& Wiley, J. L. Sex differences in cannabinoid pharmacology: a reflection of differences in the endocannabinoid system? Life Sci. 92, 476-481 (2013).

40. Craft, R. M., Wakley, A. A., Tsutsui, K. T. \& Laggart, J. D. Sex Differences in Cannabinoid 1 vs. Cannabinoid 2 Receptor-Selective Antagonism of Antinociception Produced by $\Delta 9$ Tetrahydrocannabinol and CP55,940 in the Rat. J. Pharmacol. Exp. Ther. 340, 787-800 (2012).

41. Romero, E. M. et al. Antinociceptive, behavioural and neuroendocrine effects of CP 55,940 in young rats. Brain Res. Dev. Brain Res. 136, 85-92 (2002).

42. Tseng, A. H. \& Craft, R. M. Sex differences in antinociceptive and motoric effects of 
cannabinoids. Eur. J. Pharmacol. 430, 41-47 (2001).

43. Hernandez-Avila, C. A., Rounsaville, B. J. \& Kranzler, H. R. Opioid-, cannabis- and alcoholdependent women show more rapid progression to substance abuse treatment. Drug Alcohol Depend. 74, 265-272 (2004).

44. Schepis, T. S. et al. Gender differences in adolescent marijuana use and associated psychosocial characteristics. J. Addict. Med. 5, 65-73 (2011).

45. Bassir Nia, A. et al. The relevance of sex in the association of synthetic cannabinoid use with psychosis and agitation in an inpatient population. J. Clin. Psychiatry 80, 0-0 (2019).

46. Makela, P. et al. Low doses of delta-9 tetrahydrocannabinol (THC) have divergent effects on short-term spatial memory in young, healthy adults. Neuropsychopharmacology 31 , 462-470 (2006).

47. Pope, H. G., Jr, Jacobs, A., Mialet, J. P., Yurgelun-Todd, D. \& Gruber, S. Evidence for a sex-specific residual effect of cannabis on visuospatial memory. Psychother. Psychosom. 66, 179-184 (1997).

48. Datta, U. et al. Prospects for finding the mechanisms of sex differences in addiction with human and model organism genetic analysis. Genes Brain Behav. 19, e12645 (2020).

49. Cooper, Z. D. \& Craft, R. M. Sex-Dependent Effects of Cannabis and Cannabinoids: A Translational Perspective. Neuropsychopharmacology 43, 34-51 (2018).

50. López-Gallardo, M. et al. Maternal deprivation and adolescent cannabinoid exposure impact hippocampal astrocytes, CB1 receptors and brain-derived neurotrophic factor in a sexually dimorphic fashion. Neuroscience vol. 204 90-103 (2012).

51. Tseng, A. H., Harding, J. W. \& Craft, R. M. Pharmacokinetic factors in sex differences in Delta 9-tetrahydrocannabinol-induced behavioral effects in rats. Behav. Brain Res. 154, 77-83 (2004).

52. Gilman, J. M. et al. Cannabis use is quantitatively associated with nucleus accumbens and amygdala abnormalities in young adult recreational users. J. Neurosci. 34, 5529-5538 
(2014).

53. McQueeny, T. et al. Gender effects on amygdala morphometry in adolescent marijuana users. Behav. Brain Res. 224, 128-134 (2011).

54. Lupica, C. R., Riegel, A. C. \& Hoffman, A. F. Marijuana and cannabinoid regulation of brain reward circuits. Br. J. Pharmacol. 143, 227-234 (2004).

55. Kolb, B., Gorny, G., Limebeer, C. L. \& Parker, L. A. Chronic treatment with Delta-9tetrahydrocannabinol alters the structure of neurons in the nucleus accumbens shell and medial prefrontal cortex of rats. Synapse 60, 429-436 (2006).

56. Azad, S. C. et al. Circuitry for associative plasticity in the amygdala involves endocannabinoid signaling. J. Neurosci. 24, 9953-9961 (2004).

57. Marsicano, G. \& Lafenêtre, P. Roles of the endocannabinoid system in learning and memory. Curr. Top. Behav. Neurosci. 1, 201-230 (2009).

58. Moreira, F. A. \& Lutz, B. The endocannabinoid system: emotion, learning and addiction. Addict. Biol. 13, 196-212 (2008).

59. Goodman, J. \& Packard, M. G. The influence of cannabinoids on learning and memory processes of the dorsal striatum. Neurobiol. Learn. Mem. 125, 1-14 (2015).

60. Pintori, N. et al. Repeated exposure to JWH-018 induces adaptive changes in the mesolimbic and mesocortical dopaminergic pathways, glial cells alterations, and behavioural correlates. Br. J. Pharmacol. 178, 3476-3497 (2021).

61. Hall, W. \& Degenhardt, L. Cannabis use and the risk of developing a psychotic disorder. World Psychiatry 7, 68-71 (2008).

62. World Health Organization. Health and Social Effects of Nonmedical Cannabis Use (The). (World Health Organization, 2016).

63. Albaugh, M. D. et al. Association of Cannabis Use During Adolescence With Neurodevelopment. JAMA Psychiatry (2021) doi:10.1001/jamapsychiatry.2021.1258.

64. McGrath, J. et al. Association between cannabis use and psychosis-related outcomes using 
sibling pair analysis in a cohort of young adults. Arch. Gen. Psychiatry 67, 440-447 (2010).

65. Large, M., Sharma, S., Compton, M. T., Slade, T. \& Nielssen, O. Cannabis use and earlier onset of psychosis: a systematic meta-analysis. Arch. Gen. Psychiatry 68, 555-561 (2011).

66. French, L. et al. Early Cannabis Use, Polygenic Risk Score for Schizophrenia and Brain Maturation in Adolescence. JAMA Psychiatry 72, 1002-1011 (2015).

67. Zuo, Y. et al. Unveiling the Pathogenesis of Psychiatric Disorders Using Network Models. Genes 12, (2021).

68. Gobbi, G. et al. Association of Cannabis Use in Adolescence and Risk of Depression, Anxiety, and Suicidality in Young Adulthood: A Systematic Review and Meta-analysis. JAMA Psychiatry 76, 426-434 (2019).

69. Volkow, N. D. et al. Brain glucose metabolism in chronic marijuana users at baseline and during marijuana intoxication. Psychiatry Res. 67, 29-38 (1996). 